\title{
Difficulty of internal bond prediction of particleboard using the density profile
}

Hideaki Korai ${ }^{*}$ (i)

\begin{abstract}
The relationship between density profile and internal bond (IB) of commercial particleboards was investigated. Minimum density was theoretically related to the IB, but the correlation coefficient between them was low at 0.435 . The correlation coefficients between core layer densities and IB were also low. These correlation coefficients were approximately 0.460 . The IB is influenced not only by density, but also by other factors such as the manufacturing conditions. In addition, commercial particleboards have a narrow density range. This narrow density range results in overfitting, showing a low correlation coefficient. Thus, predicting the IB using density profile was difficult.
\end{abstract}

Keywords: Particleboard, Density profile, Internal bond, Minimum density, Overfitting

\section{Introduction}

Most researchers have not studied the mechanical properties of commercial particleboard (i.e., board) but have studied those of laboratory boards. Therefore, the mechanical properties of commercial boards should be properly investigated. In particular, the relationship between density profile and internal bond (IB) should be studied although density profile is one of the important factors for IB prediction [1-3]. The minimum density in the core layer is theoretically the most important to correctly predict IB [4]. However, Schulte and Frühwald [5] showed that the correlation coefficient between minimum density and IB was not high. They described that the IB shows no correlation to the minimum density for three possible reasons [5]: (1) the measured density profile at a given location is not representative of the entire specimen. The measurement slot length is only $10 \mathrm{~mm}$ versus the $50 \mathrm{~mm}$ specimen height. (2) The failure surface is not always plane and parallel to the specimen surface. (3) The IB depends more on other parameters than on the density profile.

Their study [5] was commenced in 1996, and the recent density profiler has improved since 1996. The

\footnotetext{
*Correspondence: korai@ffpri.affrc.go.jp

Forestry and Forest Products Research Institute, Tsukuba 305-8687, Japan
}

measurement slot length of the recent density profiler was $50 \mathrm{~mm}$ versus the $50 \mathrm{~mm}$ specimen height, representative of the entire specimen. The first reason was solved. The author also often observed the second reason, and therefore, measuring the failure position was very difficult. Thus, the failure position was not determined in the present study. Whether the third reason is correct or not should be confirmed. The relationship between density profile and IB in the present study confirmed the third reason.

Previous studies $[6,7]$ have shown that the modulus of rupture (MOR) prediction using density profile was possible. The present study also established whether a density profile may be used to predict IB. These previous $[6,7]$ and present studies are the first steps toward investigating the relationships between the mechanical properties and density profile of commercial wood-based boards, including fiberboards. The future goal of these studies is to predict mechanical properties using density profile and non-destructive test. The manufacturing conditions of commercial boards, in general, are not revealed owing to industrial secrets; therefore, analyzing manufacturing conditions is impossible. However, measuring density profile and using non-destructive test are possible; therefore, a series of these studies focused on density 
profile and non-destructive test. In particular, the present study focused on just the density profile.

\section{Experimental}

\section{Density profile and IB measurement}

Commercial boards bonded with methylene diphenyl diisocyanate resin fabricated in a Japanese factory were used with the thickness and density of $9.1 \mathrm{~mm}$ and $0.78 \mathrm{~g} /$ $\mathrm{cm}^{3}$, respectively. According to Japanese industrial standard (JIS) A 5908:2003 [8], these boards were categorized as type 18 . Many MOR $50 \mathrm{~mm} \times 210 \mathrm{~mm}$ specimens were prepared from some commercial boards. These commercial boards were obtained from the same lot. Moreover, 127 MOR specimens were randomly obtained from many MOR specimens. The MOR was determined according to JIS A 5908:2003 [8].

The two density profile specimens were obtained from one MOR specimen after MOR measurements [6, 7]. Two adjacent $50 \mathrm{~mm} \times 50 \mathrm{~mm}$ specimens (specimens A and $B$ ) were derived. In addition, 127 pairs of specimens $\mathrm{A}$ and $\mathrm{B}$ existed. Figure 1 shows their sampling positions within the MOR specimen. These specimens were scanned using a density profile measuring system (DA-X 5000, GreCon, Germany). The measurement slot length of the density profiler was $50 \mathrm{~mm}$ versus the $50 \mathrm{~mm}$ specimen height. The density was measured at intervals of $0.1 \mathrm{~mm}$ along with the specimen thickness. After density profile measurements, the IB of specimens $A$ and $B$ was determined according to JIS A 5908:2003 [8].

\section{Definition of the layer densities in the density profile}

A density profile specimen was randomly selected, No. 41 specimen A, shown in Fig. 2a. Moreover, Fig. 2b shows the mean density profile of 127 specimens A and 127 specimens B (the sum was 254 specimens), and how each layer was defined in the density profile. The left layers LL1-LL3 and right layers RL1-RL3 were manufactured with fine particles and identified the face layers (0-1.7 and 7.4-9.1 mm thicknesses), whereas the left layers LL4-LL8, right layers RL4-RL8, and center layer L9 were manufactured with coarse particles and identified the core layers (1.7-7.4 mm thickness) [9]. The definition of the layer densities (Fig. 2b) was used

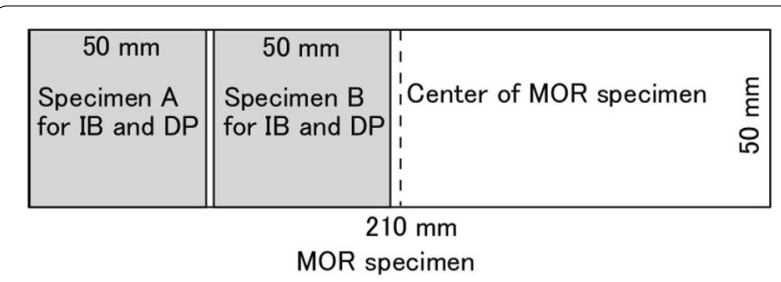

Fig. 1 Sampling positions of specimens $A$ and $B$ in the modulus of rupture (MOR) specimen. IB internal bond, DP density profile

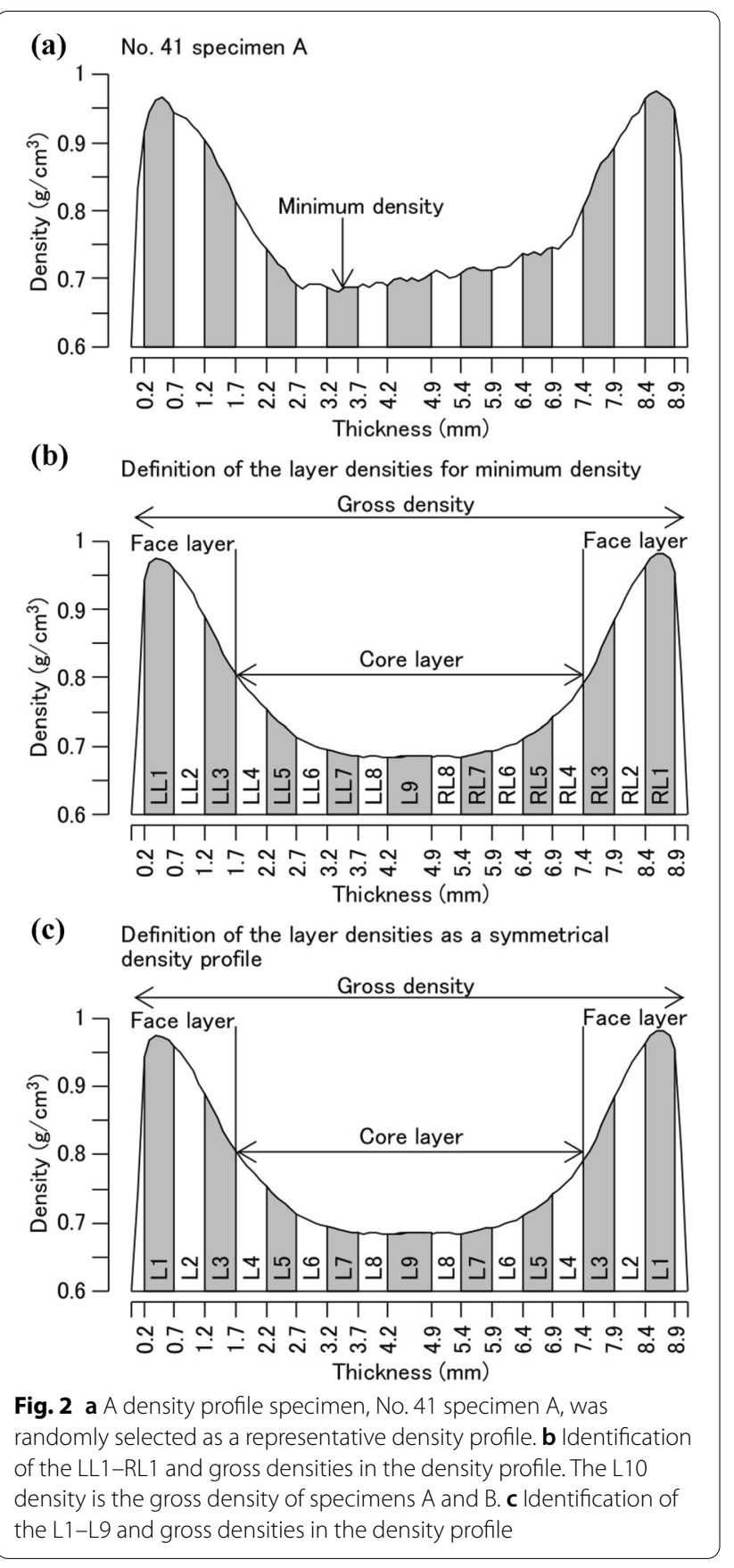

for determining the minimum density. The mean density of each layer was calculated. For example, the LL1 density was obtained by averaging the values measured at $0.2-0.7 \mathrm{~mm}$. The gross density of specimens $\mathrm{A}$ and $\mathrm{B}$ was also measured. Fig. 2c also shows the mean density profile of 254 specimens. The difference between Fig. $2 b$ and $c$ was the definition of the layer densities. For example, LL1 and RL1 densities (Fig. 2b) were obtained by averaging the density measured at $0.2-0.7$ 
and 8.4-8.9 $\mathrm{mm}$ thicknesses, respectively. The L1 density (Fig. 2c) was obtained by averaging the densities measured at $0.2-0.7$ and $8.4-8.9 \mathrm{~mm}$ thicknesses.

\section{Results and discussion}

IB of the adjacent specimens A and B

Figure $3 a$ and $b$ shows the histogram of 127 IB values of the adjacent specimens A and B, respectively. The IB distribution is symmetrical in general [10], but asymmetrical in this case. Furthermore, the MOR distribution obtained from the identical specimen (Fig. 1) was also symmetrical in the other study. Because the source of this asymmetry is unknown, more research on different boards is required. The mean, standard deviation, and the distributions of specimens A and B were nearly identical. Moreover, 127 pairs of IB (specimens A and B) existed. According to the paired $t$-test, the $p$ value was 0.571 . No statistically significant difference was observed between specimens A and B. Specimen B was obtained near the center of the MOR specimen after MOR measurement. Although there is a possibility that the IB of specimen $B$ decreased compared with that of specimen $A$ owing to the destructive effect of MOR measurement, the IB of specimens A and B was almost the same. No destruction effect on the IB decrease of specimen $B$ was found for the MOR measurement. Hence, specimens $A$ and $B$ were not distinguished. In addition, Fig. 3c shows the histogram of 254 IB values obtained from specimens A and B. Furthermore, 254 IB values were analyzed in the next section.

\section{Relationship between layer densities and IB}

The minimum density of the specimens was investigated because IB is theoretically related to minimum density [4]. L9 is assumed to be the minimum density because L9, shown in Fig. 2b, was the center of the specimen thickness. However, the number of the specimen with the minimum density was only 49 , and 205 other specimens were not L9. L9 was not always the minimum density. Figure 4a shows the relationship between minimum density and IB. The correlation coefficient was 0.435 , which was low. Furthermore, minimum density was found not to be a decisive factor to determine IB. Sequentially, the definition of the layer densities shown in Fig. 2c was used to investigate the relationship between layer densities and IB. The density profile was symmetrical, and therefore, distinguishing the right and left layers was meaningless. Accordingly, the right and left layers were equally treated in Fig. 2c. Figure $4 \mathrm{~b}-\mathrm{k}$ shows the relationship between layer densities and IB. The lowest correlation coefficient was 0.208 of the L1 density (Fig. 4b), and the highest correlation coefficient was 0.506 of the gross density (Fig. 4k). L1 was especially very low. L1 was the outermost layer, and therefore, L1 density was affected with sanding. The local surface was sanded deeply or shallowly, resulting in large dispersion of L1 density. This leads to the lowest correlation coefficient.

The failure position of the IB, in general, is probably L6-L9. This is considered to lead to high correlation coefficients of L6-L9. However, these were low. When comparing L2-L3 (L1 was excluded owing to effects of sanding) and L6-L9, not much difference was observed between their correlation coefficients. Figure 5 shows the relationship between thickness and mean layer density. The L6-L9 densities were almost the same, and therefore,
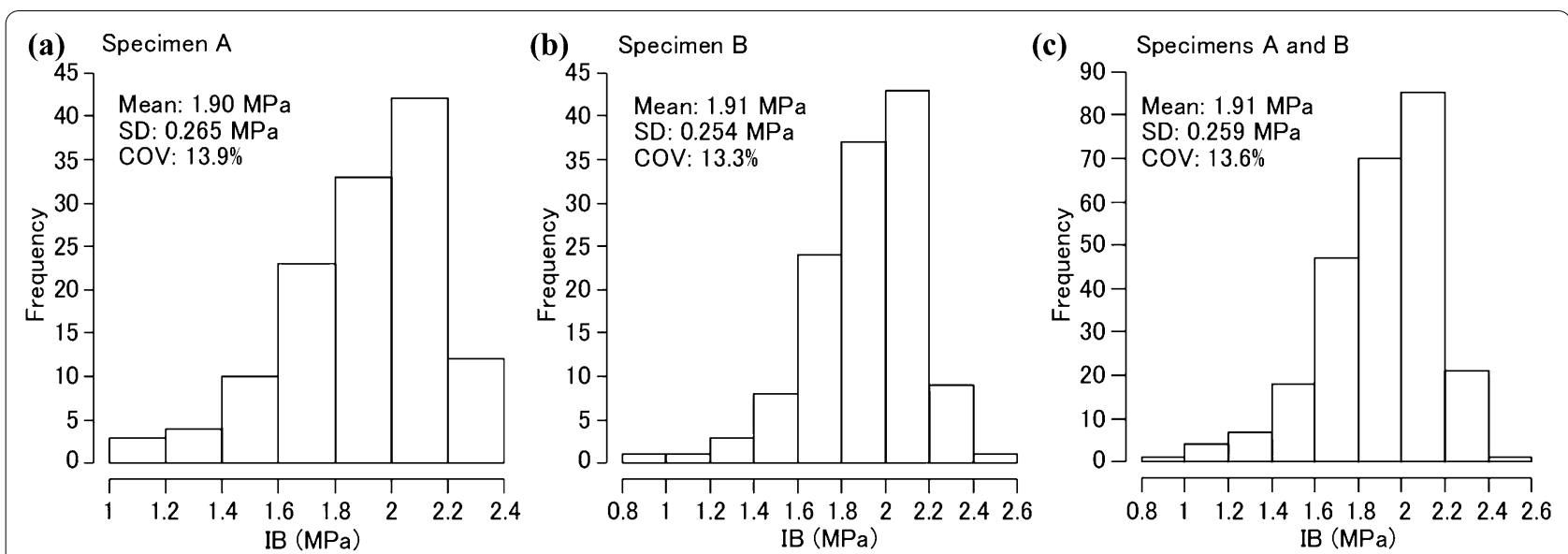

Fig. 3 a Histogram of 127 internal bond (IB) values of specimen A. b Histogram of 127 IB values of specimen B. $\mathbf{c}$ Histogram of 254 IB values of the adjacent specimens $\mathrm{A}$ and $\mathrm{B}$. SD standard deviation, COV coefficient of variation 

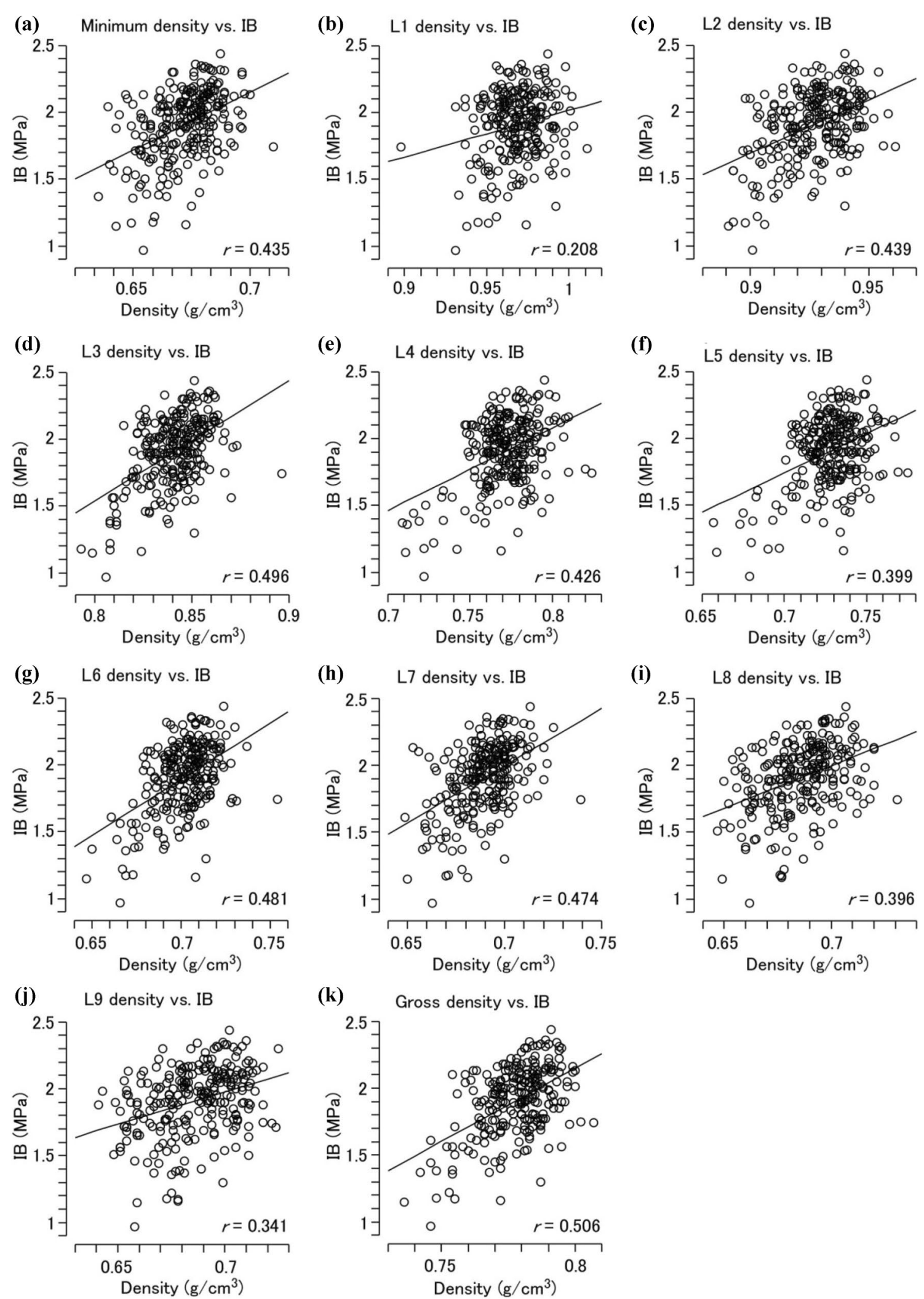

Fig. 4 The relationship between the layer densities and internal bond (IB). a Minimum density vs. IB. $\mathbf{b} L 1$ density vs. IB. $\mathbf{c} L 2$ density vs. IB. $\mathbf{d} L 3$ density vs. IB. e $\angle 4$ density vs. IB. $\mathbf{f} L 5$ density vs. IB. $\mathbf{g} L 6$ density vs. IB. $\mathbf{h} L 7$ density vs. IB. $\mathbf{i} L 8$ density vs. IB. $\mathbf{j} L 9$ density vs. IB. $\mathbf{k}$ Gross density vs. IB 


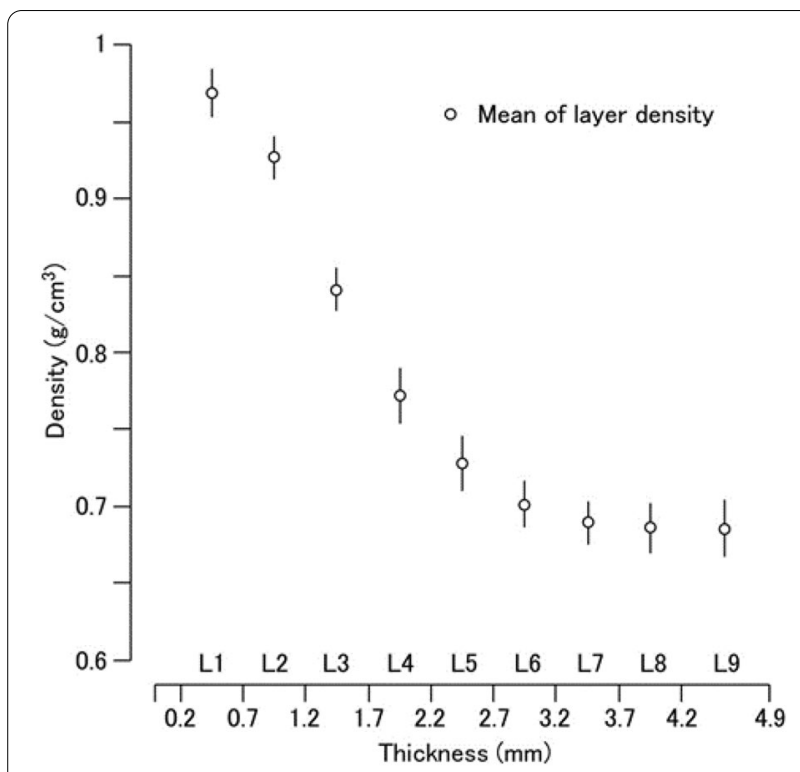

Fig. 5 Relationship between thickness and mean layer density. Error bars denote standard deviations

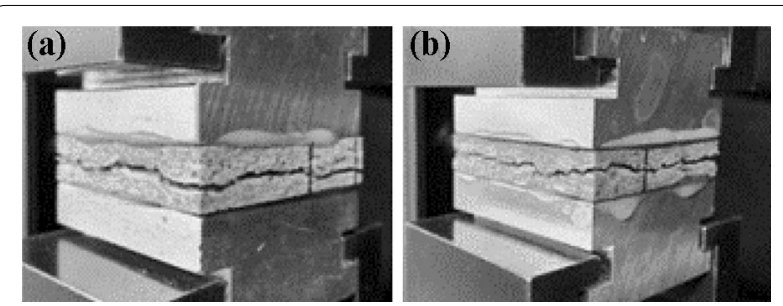

Fig. 6 Failure positions of two randomly selected IB specimens. a Randomly selected No. 35 specimen A. b Randomly selected No. 86 specimen $A$ the difference between minimum density and L6-L9 densities was subtle (e.g., No. 41 specimen A shown in Fig. 2a). Furthermore, the failure surface is not always plane and parallel to the specimen surface (Fig. 6); therefore, the failure position cannot be defined at layers as shown in Fig. 2b. Thus, layer density was not a decisive factor in predicting IB. Rather than L1-L9 and minimum density, gross density is more important. Schulte and Frühwald [5] also showed that gross density is a better predictor of IB than minimum density [5]. Their correlation coefficients of minimum and gross densities were $0.24-0.59$ and $0.46-0.83$, respectively [5]. The correlation coefficients of minimum and gross densities of the present study were 0.435 and 0.506 , respectively (Fig. $4 \mathrm{a}$ and k).

These layer densities as shown in Fig. 2, that is, density profile, were not useful to predict IB. Moreover, the IB is influenced not only by density profile, but also by other factors such as the manufacturing conditions. This is why making an IB prediction using density profile is difficult.

\section{Difference between laboratory and commercial boards}

Several studies have shown the high correlation coefficient between density and IB, indicating the importance of density. For example, Wang et al. made boards at density ranging from 0.3 to $1.1 \mathrm{~g} / \mathrm{cm}^{3}$ [11]. Jin et al. also made strand boards at different density ranging from 0.4 to $0.9 \mathrm{~g} / \mathrm{cm}^{3}$ [12]. Their results showed a high correlation between density and IB. The coefficients of determination of Wang et al. [11] and Jin et al. [12] were approximately 0.99 and 0.97 , respectively. From the aforementioned similar studies [11, 12], density was considered to be a decisive factor to predict IB. This fact resulted from laboratory boards having a wide density range, thus proving the importance of density. However, commercial boards do not have a wide density range, and the density range was very narrow, as shown in Fig. 4. The gross density range in the present study was only $0.742-0.807 \mathrm{~g} / \mathrm{cm}^{3}$ (Fig. $4 \mathrm{k}$ ). This narrow density range results in overfitting, showing a low correlation coefficient. Thus, density and density profile are not important for IB prediction of commercial boards that have a narrow density range.

Dai et al. [13, 14] modeled some of the essential aspects of wood composites bonding. Their works set a stage for the development of a comprehensive model to predict IB. However, their model was developed from wide-range density conditions as described above. A model should be developed from narrow-range density conditions when the IB of the commercial board is predicted.

\section{Difficulty of IB prediction using density profile}

MOR and IB specimens were obtained from the identical board piece as shown in Fig. 1. MOR prediction using density profile was possible in the previous studies $[6,7]$. The correlation coefficient between L4 density and MOR was 0.610 . However, IB prediction using a density profile was impossible in the present study; the correlation coefficients shown in Fig. 4 were lower than 0.610. Thus, the effects of density profile on MOR and IB predictions differ entirely. To deepen these analyses, Fig. 7 shows the relationship between IB and MOR. These correlation coefficients of specimens A and B were 0.270 and 0.241 , respectively, which were very low. IB prediction was also difficult using MOR.

The IB is influenced not only by density profile, but also by manufacturing conditions, such as the type of glue, glue content, particle dimensions, wood species, relative densification (concerning wood density), moisture 

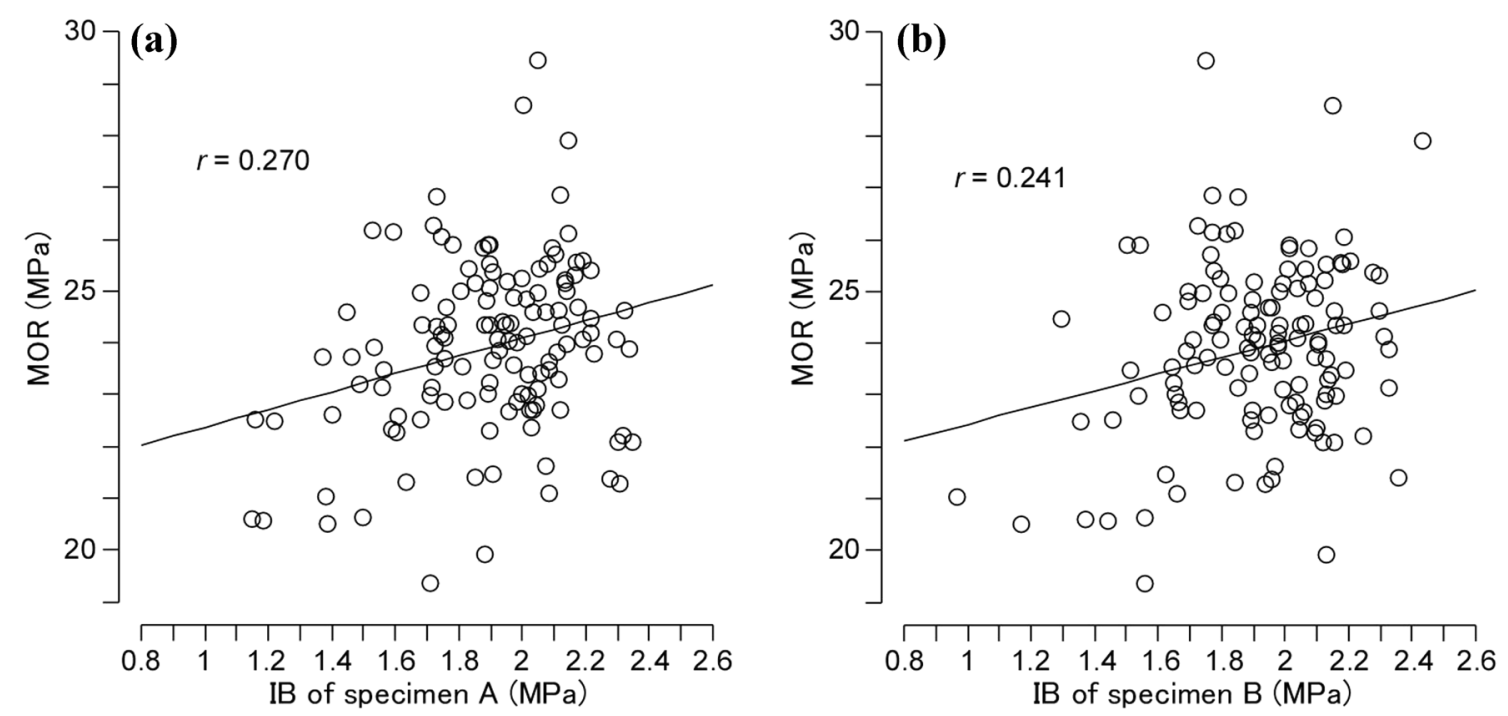

Fig. 7 a The relationship between internal bond (IB) of specimen A and modulus of rupture (MOR). $\mathbf{b}$ The relationship between IB of specimen $B$ and MOR. r: correlation coefficient

content of the mat, and pressing parameter [5]. These factors are very difficult to analyze. Moreover, overfitting results in a difficult IB prediction using density profile.

\section{Conclusions}

The correlation coefficients between layer densities including minimum density and IB, were low. These correlation coefficients were approximately 0.460 . Correctly predicting the IB using density profile was found to be very difficult. The IB is influenced not only by density, but also by other factors such as manufacturing conditions. Recently, many studies reported that a high correlation coefficient between density and IB was observed. This fact resulted from laboratory boards with a wide density range. However, commercial boards have a narrow density range, resulting in overfitting, thereby producing a low correlation coefficient.

\footnotetext{
Abbreviations

IB: Internal bond; MOR: Modulus of rupture; JIS: Japanese Industrial Standards; L1: Layer 1; L2: Layer 2; L3: Layer 3; L4: Layer 4; L5: Layer 5; L6: Layer 6; L7: Layer 7; L8: Layer 8; L9: Layer 9; LL1: Left layer 1; LL2: Left layer 2; LL3: Left layer 3; LL4: Left layer 4; LL5: Left layer 5; LL6: Left layer 6; LL7: Left layer 7; LL8: Left layer 8; L9: Center layer; RL1: Right layer 1; RL2: Right layer 2; RL3: Right layer 3; RL4: Right layer 4; RL5: Right layer 5; RL6: Right layer 6; RL7: Right layer 7; RL8: Right layer 8.
}

\section{Acknowledgements}

The author expresses the gratitude to Mr. Kazuo Hattori and Mr. Takashi Fuchigami of Japan Novopan Industrial Co., Ltd. for providing the particleboards. The author also expresses the gratitude to Mr. Atsushi Miyatake of Forestry and Forest Products Research Institute for his kind suggestions.
Authors' contributions

HK designed and conducted the study. The author read and approved the final manuscript.

\section{Funding}

Not applicable.

\section{Availability of data and materials}

Not applicable.

\section{Declarations}

\section{Competing interests}

The author declares that they have no competing interests.

Received: 9 June 2021 Accepted: 19 October 2021

Published online: 06 November 2021

References

1. Strickler MD (1959) Effect of press cycle and moisture content on properties of Douglas-Fir flakeboard. For Prod J 7:203-215

2. Kelly MW (1977) Critical literature review of relationships between processing parameters and physical properties of particleboard, USDA Forest Service Forest Products Laboratory General Technical Report FPL-10. pp $4-15$

3. Suo S, Bowyer JL (1994) Simulation modeling of particleboard density profile. Wood Fiber Sci 26:397-411

4. Kawai S, Sasaki H (1986) Production technology for low-density particleboard I. Forming a density gradient and its effect on board properties. Mokuzai Gakkaishi 32:324-330

5. Schulte M, Frühwald A (1996) Some investigations concerning density profile, internal bond and relating failure position of particleboard. Holz Roh Werkst 54:289-294

6. Korai H (2020) Importance of core layer density to predict the bending strength of particleboard (in Japanese). In: Abstracts of the 38th annual meeting of Wood Technological Association of Japan, Gifu, October 2020

7. Korai $\mathrm{H}$ (2021) Effects of core layer density on bending strength of particleboard (in Japanese). In: Abstracts of the 71th annual meeting of 
the Japan Wood Research Society, Tokyo University of Agriculture and Technology, Fuchu, 19-21 March 2021

8. Japanese Industrial Standards (2003) JIS standard specification for particleboard. JIS A 5908. Japanese Standards Association, Tokyo

9. Stark NM, Cai Z, Carll C (2010) Wood-Based Composite Materials Panel Products, Glued-Laminated Timber, Structural Composite Lumber, and Wood-Nonwood Composite Materials. Wood handbook—wood as an engineering material.: USDA Forest Service, Forest Products Laboratory, General Technical Report, FPL-GTR-190, Chapter 11, p11

10. Korai H, Watanabe K (2019) Bayesian Analysis of strength properties of particleboard (in Japanese). Mokuzai Gakkaishi 65:93-101

11. Wong ED, Zhang M, Wang Q, Kawai S (1999) Formation of density profile and its effects on properties of particleboard. Wood Sci Tech 33:327-340
12. Jin J, Dai C, Hsu WE, Yu C (2009) Properties of strand boards with uniform and conventional vertical density profiles. Wood Sci Tech 43:559-574

13. Dai C, Yu C, Zhou C (2007) Theoretical modeling of bonding characteristics and performance of wood composites. Part I Inter-element contact. Wood Fiber Sci 39:48-55

14. Dai C, Yu C, Jin J (2008) Theoretical modeling of bonding characteristics and performance of wood composites. Part IV. Internal bond strength. Wood Fiber Sci 40:146-160

\section{Publisher's Note}

Springer Nature remains neutral with regard to jurisdictional claims in published maps and institutional affiliations.

\section{Submit your manuscript to a SpringerOpen ${ }^{\circ}$ journal and benefit from:}

- Convenient online submission

- Rigorous peer review

- Open access: articles freely available online

- High visibility within the field

- Retaining the copyright to your article

Submit your next manuscript at $\gg$ springeropen.com 